\title{
On the application of the PFEM to droplet dynamics modeling in fuel cells
}

\author{
Pavel B. Ryzhakov ${ }^{1,2}$ - Alex Jarauta ${ }^{2} \cdot$ Marc Secanell $^{3} \cdot$ Jordi Pons-Prats $^{2}$
}

Received: 2 March 2016 / Revised: 7 April 2016 / Accepted: 8 April 2016 / Published online: 26 April 2016

(C) OWZ 2016

\begin{abstract}
The Particle Finite Element Method (PFEM) is used to develop a model to study two-phase flow in fuel cell gas channels. First, the PFEM is used to develop the model of free and sessile droplets. The droplet model is then coupled to an Eulerian, fixed-grid, model for the airflow. The resulting coupled PFEM-Eulerian algorithm is used to study droplet oscillations in an air flow and droplet growth in a lowtemperature fuel cell gas channel. Numerical results show good agreement with predicted frequencies of oscillation, contact angle, and deformation of injected droplets in gas channels. The PFEM-based approach provides a novel strategy to study droplet dynamics in fuel cells.
\end{abstract}

Keywords PFEM - Embedded model · Fuel cells · Droplet dynamics $\cdot$ Sessile droplet

\section{Introduction}

Application of interest: fuel cells Most conventional energy conversion devices rely on the combustion of fossil fuels, such as oil or coal, to produce power resulting in greenhouse gases, particulates, and other negative pollutants. Polymer electrolyte fuel cells provide an alternative energy conversion device that, when powered with hydrogen, produces zeroemission and operates quietly and with a higher efficiency. In order to increase their market penetration, however, the cost

Pavel B. Ryzhakov

pryzhakov@cimne.upc.edu

1 Centre Internacional de Métodes Numérics en Enginyeria (CIMNE), Gran Capitán s/n, 08034 Barcelona, Spain

2 CIMNE, Barcelona, Spain

3 Energy Systems Design Lab (ESDLab), University of Alberta, Edmonton, Canada of fuel cell should be substantially reduced. Cost reductions can be achieved by increasing the maximum current produced in a fuel cell, which is usually limited by the ability of the fuel cell to remove liquid water from the gas diffusion electrode and the channels. In an operating hydrogen polymer electrolyte fuel cell (PEFC), water is produced by the oxygen reduction reaction (ORR) in the cathode catalyst layer (CL). At high current densities, excess liquid water is evacuated through the pores of the gas diffusion layer (GDL). When it emerges from the pores into the gas channels it may form droplets, films, or slug flows depending on the working conditions [5] (Fig. 1). Film formation can severely limit the area of the fuel cell available to deliver reactants to the reaction sites. Slug flow might lead to flow malfunction where some channels in the cell do not receive fresh oxygen thereby de-activating large regions of the cell [24]. Optimal water removal from the channels is therefore critical.

Two-phase flow in micro-channels The channels encountered in fuel cells are of sub-millimeter size. Channels of this size are known as micro-channels [36]. Analysis of twophase flow in micro-channels is an active area of research $[2,4,12,14,36]$. Apart from fuel cell, such settings are found in other important applications including micro-reactors and micro-heat exchangers. In such systems, one deals with the interaction of either airflow with liquid droplets or liquid flow with gas bubbles. In order to predict the behavior of such systems, it is of paramount importance to model each of the phases and their interaction at the interface. Dominant effects depend on the considered scale. Surface tension and viscous effects are usually more important than gravitational or inertial effects within micro-channels. These latter effects, however, cannot be neglected. At the considered length scale (i.e., droplets with less than $1 \mathrm{~mm}$ height), they might have a significant impact on droplet dynamics [36]. 


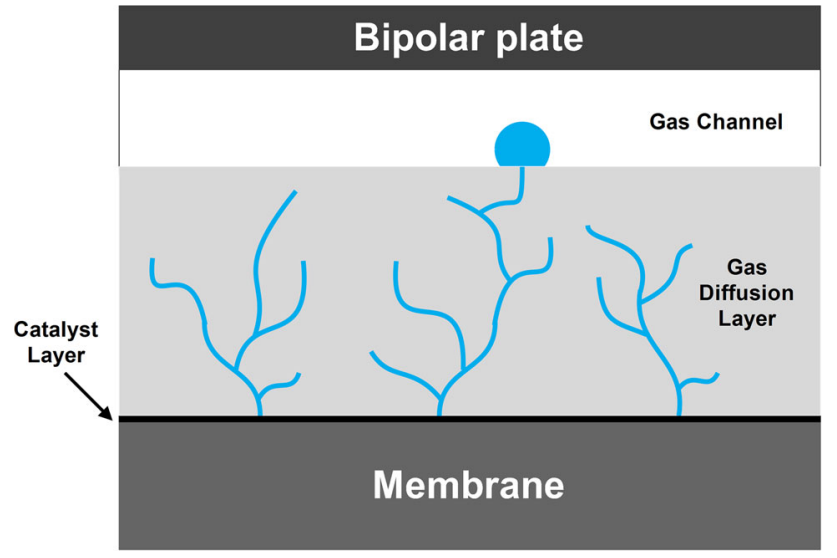

Fig. 1 Exceeding water produced in the membrane diffuses through the GDL and emerges into the gas channel as droplets, films, or slugs

Modeling challenges Interface conditions between the channel and the GDL remain largely unknown and continue to be a very active area of research [35]. Modeling of droplet dynamics in fuel cell gas channels has been studied using both analytical, e.g., [6,7,22], and numerical, e.g., [17,34,38], approaches. Most numerical studies have used either volume of fluid (VOF), e.g., [17,34,38], or level set (LS), e.g., $[1,8]$, methods. These fixed-grid (Eulerian) formulations can theoretically handle arbitrary large deformations since the mesh is fixed and elements do not deteriorate. However, the advective term is difficult to discretize due to the jump of the volume fraction function at the interface [17]. Artificial diffusion of the interface is also observed in the solution when coarse meshes are used at the vicinity of the interface. These Eulerian models typically require large computational times due to the challenges of surface tension modeling on fixed meshes (see e.g., [32]). Surface tension modeling in Eulerian approaches is challenging since the interface is not explicitly represented by the mesh in such approaches. Thus, the surface tension force which is actually a surface force is often represented as a force acting at a layer of finite thickness. Moreover, accurate representation of the discontinuity in the pressure and pressure gradient across the air-water interface requires additional techniques (such as shape function enrichments) whenever fixed mesh approaches are used.

The Particle Finite Element Method (PFEM) Particle Finite Element Method (PFEM) provides an alternative methodology to tackle two-phase flow in micro-channels. Proposed by Idelsohn et al. [19] and Oñate et al. [29], the method is a combination of a particle approach and the finite element method. The domain is represented by a set of particles that move in a Lagrangian manner according to the velocity field [28]. Forces are then obtained using a background mesh, where the nodes of this mesh coincide with the particles. Since all the information is stored in the particles, the PFEM does not exhibit numerical diffusion due to re-meshing. For droplet-airflow interaction problems, such as droplet-airflow interaction, PFEM may be used for both the liquid and the gas domains. The interface becomes explicitly represented by the mesh and no interface smearing often observed in Eulerian approaches takes place. However, steady-state solutions using PFEM for both sub-domains usually exhibit spurious velocities at the interface due to large pressure gradients [28]. Kamran et al. [23] proposed a degree of freedom duplication to address this issue. An additional disadvantage of a purely PFEM-based approach for droplet-air systems is that it would require re-meshing the entire domain (i.e., both the droplet and the air) at each time step, which may be computationally tedious. Novel particle-based formulations that exclude the deforming Lagrangian meshes (and allow for using large time steps) have been recently proposed [13]. However, this approach is not advantageous for the problem at hand as they preclude mesh-based boundary/interface representation beneficial for the surface tension computations.

A numerical method that combines the advantages of both Eulerian and the PFEM formulations is the PFEM-Eulerian numerical approach. In this approach, the droplet, modeled using the PFEM, moves on top of a fixed Eulerian mesh (representing the air). This approach falls into the class of "embedded" or "immersed boundary methods" widely used in the field of fluid-structure interaction (FSI) modeling $[15,16,25]$. For multi-fluid and multi-phase problems, this approach has been introduced only recently. Conceptually, the embedded framework for multi-phase analysis was proposed in [30, Chap. 5] and [27], and extended to account for surface tension in [31] and [21].

In this article, the PFEM-Eulerian is presented as an alternative method to the traditional Eulerian approaches to study the dynamics of liquid water droplets emerging from the gas diffusion electrode to the gas channel of a polymer electrolyte fuel cell (PEFC). The method naturally tracks the interface, thereby mitigating the issues with previous Eulerian formulations, such as artificial diffusion of the interface and high computational time. This approach allows to accurately track the droplet domain boundaries so that boundary conditions and forces (such as surface tension) can be directly applied to interface nodes. Additional techniques used in purely Eulerian (fixed-grid) approaches, where boundaries and interfaces cut the computational mesh at arbitrary positions, are therefore not necessary. The method is shown to be capable to study both droplet deformation and shedding, as well as droplet growth at different flow rates.

The article is organized as follows. First, the numerical model for the two-phase flow is presented. The droplet model is validated in both two and three dimensions. The model is then used to analyze the behavior of a sessile droplet in different substrates with variable contact angle. Droplet dynamics in a fuel cell gas channel are studied next. Droplet dynamics 
simulation in gas channel accounting for variable volumes is performed.

\section{Numerical model}

Let us consider Lagrangian domain $\Omega_{\mathrm{L}}$ (water droplet) embedded into the Eulerian domain $\Omega_{\mathrm{E}}$ (air) with an external boundary $\Gamma_{\mathrm{E}}$ (see Fig. 2). In the embedded setting, the interface $\Gamma_{\mathrm{I}}$ between the two fluids is defined by the position of the boundary of the Lagrangian domain $\Gamma_{\mathrm{L}}$. The interface $\Gamma_{\mathrm{I}}$ splits the Eulerian domain into two parts: the real one $\Omega_{\mathrm{E}}^{r}$ (representing the gas) and the fictitious one $\Omega_{\mathrm{E}}^{f}$ that does not have physical meaning. Note that at the continuous level the fictitious Eulerian domain exactly coincides with the Lagrangian domain and $\Gamma_{\mathrm{I}}=\Gamma_{\mathrm{L}}$. At the discrete level $\Gamma_{\text {I }}$ can be thought of as the polyline or polygon that is connecting the intersections between the Lagrangian boundary $\Gamma_{\mathrm{L}}$ and the Eulerian elements (see dashed line in Fig. 2b).

The governing system of equations in either domain is the Navier-Stokes equations equipped with the incompressibility condition:

$\rho \frac{D \mathbf{v}}{D t}-\nabla \cdot\left(\mu\left(\nabla \mathbf{v}+\nabla^{T}(\mathbf{v})\right)\right)+\nabla p=\rho \mathbf{g}$ in $\Omega_{\mathrm{E}}^{\mathrm{r}}$ and $\Omega_{\mathrm{L}}$

$\nabla \cdot \mathbf{v}=0$ in $\Omega_{\mathrm{E}}^{\mathrm{r}}$ and $\Omega_{\mathrm{L}}$

where $\frac{D \mathbf{v}}{D t}$ is the material time derivative of the velocity, $p$ is the pressure, $t$ is the time, $\mathbf{g}$ is the body force, $\rho$ is the fluid density, and $\mu$ is the fluid dynamic viscosity. The physical properties are defined as $\rho=\rho_{E}$ and $\mu=\mu_{E}$ in $\Omega_{\mathrm{E}}$, and $\rho=\rho_{L}$ and $\mu=\mu_{L}$ in $\Omega_{\mathrm{L}}$.

Boundary and interface conditions In order to ensure the well-posedness of the Navier-Stokes problem defined by Eqs. (1) and (2), suitable boundary conditions must be specified. On the external boundary $\Gamma_{\mathrm{E}}=\Gamma_{D} \cup \Gamma_{N}$, such that $\Gamma_{D} \cap \Gamma_{N}=\emptyset$, the following conditions are prescribed:

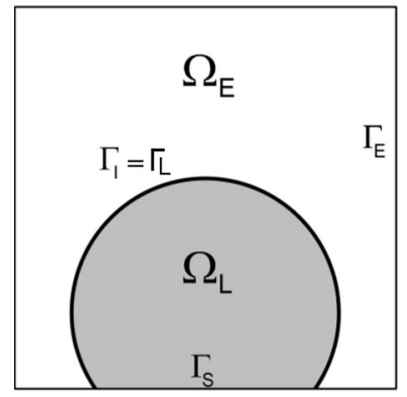

(a)

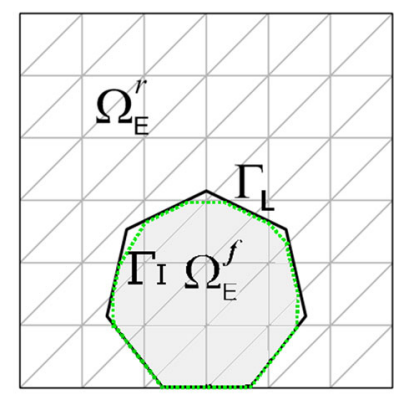

(b)
Fig. 2 Embedded setting: a continuum representation b Lagrangian boundary, its representation on the Eulerian mesh and real/fictitious sub-domains $\mathbf{v}=\mathbf{v}^{p r}$ at $\Gamma_{D}$

$\boldsymbol{\sigma} \cdot \mathbf{n}=\boldsymbol{\sigma}_{n}^{p r}$ at $\Gamma_{N}$

where $\mathbf{v}^{p r}$ is the prescribed velocity, $\mathbf{n}$ is the outer unit normal to $\Gamma_{N}$, and $\sigma_{n}^{p r}$ is the prescribed traction vector.

On the internal interface $\Gamma_{\mathrm{I}}$ the coupling conditions are

$\llbracket \mathbf{v} \rrbracket=0 \quad$ at $\quad \Gamma_{\mathrm{I}}$

$\llbracket \boldsymbol{\sigma} \rrbracket \cdot \mathbf{n}=\gamma \kappa \mathbf{n}$ at $\Gamma_{\mathrm{I}}$

where $\mathbf{n}$ now is the unit normal to the interface $\Gamma_{\mathrm{I}}, \gamma$ and $\kappa$ are the surface tension coefficient and the interface curvature, respectively. The $\llbracket x \rrbracket$ symbol represents the jump of the quantity $x$ across the interface.

Equation (5) expresses the continuity of all velocity components $\left(\llbracket \mathbf{v} \rrbracket=\mathbf{v}_{E}-\mathbf{v}_{L}\right.$, where indexes $E$ and $L$ distinguish the quantities corresponding to the air and water, respectively). The equality of the normal components of velocity ensures no mass flow across the interface. The tangential components' equality is similar to a no-slip condition and is necessary when fluids with non-zero viscosity are considered. Eq. (6) expresses that the difference in the normal stress across the interface is balanced by the surface tension force.

Projecting Eq. (6) onto the normal and tangential directions leads to the following scalar interface conditions:

$\mathbf{n} \cdot(\llbracket \boldsymbol{\sigma} \rrbracket \cdot \mathbf{n})=\gamma \kappa \quad$ at $\quad \Gamma_{\mathrm{I}}$

$\mathbf{t} \cdot(\llbracket \boldsymbol{\sigma} \rrbracket \cdot \mathbf{n})=0 \quad$ at $\quad \Gamma_{\mathrm{I}}$

Noting that the jump in the stress across the interface is equal to the difference between the stresses of the two fluids $\llbracket \sigma \rrbracket=$ $\sigma_{E}-\sigma_{L}$ and splitting the stress tensor into volumetric and deviatoric part results in:

$$
\begin{gathered}
\left(p_{L}-p_{E}\right)+\left[\mu_{E} \mathbf{n} \cdot\left(\left[\nabla \mathbf{v}+\nabla^{T} \mathbf{v}\right]_{E} \cdot \mathbf{n}\right)\right. \\
\left.-\mu_{L} \cdot \mathbf{n} \cdot\left(\left[\nabla \mathbf{v}+\nabla^{T} \mathbf{v}\right]_{L} \cdot \mathbf{n}\right)\right]=\gamma \kappa \\
\mu_{E} \mathbf{t} \cdot\left(\left[\nabla \mathbf{v}+\nabla^{T} \mathbf{v}\right]_{E} \cdot \mathbf{n}\right) \\
-\mu_{L} \cdot \mathbf{t} \cdot\left(\left[\nabla \mathbf{v}+\nabla^{T} \mathbf{v}\right]_{L} \cdot \mathbf{n}\right)=0
\end{gathered}
$$

The boundary condition on $\Gamma_{S}$ is corresponding to the contact with the solid surface and enforces the contact angle value known from experiments. It is described in sessile droplet section (Sect. 3.2).

\subsection{PFEM model for the water droplets}

Governing Eqs. (1) and (2) are discretized in space. Being standard, the FEM discretization process is not detailed here. Velocity and pressure are approximated by linear interpolation functions over 3-noded triangles in 2D or 4-noded 
tetrahedra in 3D. For sake of simplicity, Backward-Euler time discretization scheme is chosen, although any method of the $\theta$-family is valid. The problem for water domain can be stated as follows: given $\overline{\mathbf{v}}_{n}$ and $\bar{p}_{n}$ at $t_{n}$, the time discrete problem consists in finding $\overline{\mathbf{v}}_{n+1}$ and $\bar{p}_{n+1}$ (note that the nodal values are distinguished by an over-bar) at $t_{n+1}$ as the solution of:

$\mathbf{M} \frac{\overline{\mathbf{v}}_{n+1}-\overline{\mathbf{v}}_{n}}{\Delta t}+\mu \mathbf{L} \overline{\mathbf{v}}_{n+1}+\mathbf{G} \bar{p}_{n+1}=\overline{\mathbf{F}}+\overline{\mathbf{F}}_{\mathrm{st}}$
$\mathbf{D} \overline{\mathbf{v}}_{n+1}+\mathbf{S}=0$

where $\mathbf{M}$ is the mass matrix, $\mathbf{L}$ is the Laplacian matrix, $\mathbf{G}$ is the gradient matrix, $\mathbf{D}$ is the divergence matrix, $\overline{\mathbf{v}}$ and $\bar{p}$ are the velocity and pressure respectively, $\overline{\mathbf{F}}$ is the sum of the body force and the Neumann term representing the forces exerted by airflow at the droplet surface and $\overline{\mathbf{F}}_{\text {st }}$ is the surface tension force vector. The matrices are assembled from the elemental contributions, with the components defined as:

$$
\begin{aligned}
& M_{i}^{a b}=\rho_{f} \int_{\Omega_{e}} N^{a} N^{b} d \Omega \\
& L^{a b}=\int_{\Omega_{e}} \frac{\partial N^{a}}{\partial x_{i}} \frac{\partial N^{b}}{\partial x_{i}} d \Omega \\
& G_{i}^{a b}=-\int_{\Omega_{e}} \frac{\partial N^{a}}{\partial x_{i}} N^{b} d \Omega \\
& f_{i}^{a}=\rho_{f} \int_{\Omega_{e}} N^{a} g_{i} d \Omega \\
& f_{s t, i}^{a}=-\int_{\Gamma_{\mathrm{I}}} \gamma \kappa N^{a} n_{i} d \Omega \\
& D_{i}^{a b}=\int_{\Omega_{e}} N^{a} \frac{\partial N^{b}}{\partial x_{i}} d \Omega
\end{aligned}
$$

where $N^{a}$ stands for the standard linear FE shape function at node $a$ and $\Omega_{e}$ is the element integration domain. Indices $i, j$ refer to spatial components. Note that due to using a Lagrangian framework for the domain, the elemental integration domains in Eqs. (13)-(18) must be updated according to changing mesh configuration. The force $\overline{\mathbf{F}}$ in Eq. (11) includes the Neumann term due to the interaction with gas (normal and shear stress). Due to using the same degree of interpolation for velocity and pressure, Eqs. (11) and (12) must be stabilized. Discussion on stabilization techniques lies out of the scope of this work. A wide class of stabilization methods leads to introducing a stabilization Laplacian matrix of the form $\mathbf{S}=\tau \mathbf{L}$, where $\tau$ is an algorithmic stabilization coefficient defined as $\tau=\left(\frac{2|| \overline{\mathbf{v}}||}{h}+\frac{4 v}{h^{2}}\right)^{-1}$ and $h$ is the element size (see e.g., [10] for details).

Let us define the residuals of the momentum and the continuity equations (note that index $i$ stands for the non-linear iteration index):

$$
\begin{aligned}
& \overline{\mathbf{r}}_{m}=\overline{\mathbf{F}}+\overline{\mathbf{F}}_{\mathrm{st}}-\left[\mathbf{M} \frac{\overline{\mathbf{v}}_{n+1}^{i}-\overline{\mathbf{v}}_{n}}{\Delta t}+\mu \mathbf{L} \overline{\mathbf{v}}_{n+1}^{i}+\mathbf{G} \bar{p}_{n+1}^{i}\right] \\
& \overline{\mathbf{r}}_{c}=-\mathbf{D} \overline{\mathbf{v}}_{n+1}^{i}-\mathbf{S} \bar{p}_{n+1}^{i}
\end{aligned}
$$

The governing system in the residual form can thus be written as

$$
\left(\begin{array}{cc}
\frac{\mathbf{M}}{\Delta t}+\mu \mathbf{L} & \mathbf{G} \\
\mathbf{D} & \mathbf{S}
\end{array}\right)\left(\begin{array}{l}
d \overline{\mathbf{v}} \\
d \bar{p}
\end{array}\right)=\left(\begin{array}{c}
\overline{\mathbf{r}}_{m} \\
\overline{\mathbf{r}}_{c}
\end{array}\right)
$$

The details upon the implementation and linearization can be found in [31].

Once the system (Eq. 21) is solved, the velocity and the pressure are updated as $\overline{\mathbf{v}}_{n+1}^{i+1}=\overline{\mathbf{v}}_{n+1}^{i}+d \overline{\mathbf{v}}$ and $\bar{p}_{n+1}^{i+1}=$ $\bar{p}_{n+1}^{i}+d \bar{p}$. The position of the nodes in $\Omega_{\mathrm{L}}$ is updated as $\mathbf{X}_{n+1}^{i+1}=\mathbf{X}_{n}+\Delta t \cdot \overline{\mathbf{v}}_{n+1}^{i+1}$. Note that there exist different schemes for updating the nodal positions. For example, using displacement instead of velocity as a primary variable leads to "exact" nodal position update [30]. Alternatively, nodal positions can be updated using streamline integration according to [13]. The discussion of the impact of these different updating techniques upon the resulting accuracy of the overall method lies outside the scope of this work.

The integration domains necessary for the computation of the discrete operators (Eqs. 13-18) are be updated according to this new configuration $\mathbf{X}_{n+1}^{i+1}$. Discretized governing equations are solved using a monolithic scheme, where both velocity and pressure are solved simultaneously at every time step. This method is more computationally intensive, but the presence of surface tension precludes using computationally cheaper schemes such as fractional step method [31]. In the present work, surface tension is modeled implicitly. The details on the surface tension model can be found in [21].

\subsection{Eulerian formulation for the air}

Discretizing the governing Eqs. (1) and (2) in the Eulerian framework yields the following FE model for the air: ${ }^{1}$

$$
\begin{aligned}
& \mathbf{M} \frac{\overline{\mathbf{v}}_{n+1}-\overline{\mathbf{v}}_{n}}{\Delta t}+\left[\overline{\mathbf{K}}\left(\overline{\mathbf{v}}_{n+1}\right)+\mu \mathbf{L}\right] \overline{\mathbf{v}}_{n+1}+\mathbf{G} \bar{p}_{n+1}=\overline{\mathbf{F}} \\
& \mathbf{D} \overline{\mathbf{v}}_{n+1}=0
\end{aligned}
$$

where $\overline{\mathbf{K}}\left(\overline{\mathbf{v}}_{n+1}\right)$ is the non-linear convection operator, and the rest of terms have been already defined in Sect. 2.1. The convection operator is assembled from the elemental contribution, with the components defined as:

\footnotetext{
${ }^{1}$ Once again, linear pressure-velocity interpolations have been used for the space discretization while Backward Euler has been applied for the time integration.
} 
$K^{a b}=\rho_{f} \int_{\Omega_{e}} N^{a}\left(\overline{\mathrm{v}}_{i} \frac{\partial N^{b}}{\partial x_{i}}\right) d \Omega$

Pressure and convection stabilization terms are omitted here for the sake of brevity. They can be found in [31]. For reducing the computational cost associated to the solution of the governing system, the fractional splitting is applied $[9,11,18,33]$. For more detailed information on the model, as well as the coupling strategy between both domains $\Omega_{\mathrm{E}}$ and $\Omega_{\mathrm{L}}$, the reader is referred to [31].

\subsection{Coupling strategy}

The steps of the coupling strategy representing airflowdroplet interaction can be summarized as follows:

1. Solve the water problem using Eq. (21) and update the corresponding mesh position.

Output: new position of the Lagrangian mesh, $\overline{\mathbf{v}}_{n+1} \bar{p}_{n+1}$ in $\Omega_{\mathrm{L}}^{n+1}$

2. Identify the position of Lagrangian domain within the Eulerian one.

3. Fix the velocity to the velocity of the droplet surface at $\Gamma_{\mathrm{I}}$ (see [31] for further details). ${ }^{2}$

4. "Switch off" the elements corresponding to the fictitious part of the Eulerian domain $\left(\Omega_{E_{f}}\right)$.

5. Solve the gas problem (using real part of the Eulerian domain $\Omega_{E_{r}}$ ).

Output: velocity and pressure $\overline{\mathbf{v}}_{n+1}$ and $\bar{p}_{n+1}$ in $\Omega_{\mathrm{E}}^{n+1}$.

6. Project the air stresses onto the liquid boundary $\Gamma_{\mathrm{L}}$ surface and compute the corresponding force term for the momentum equation of the liquid (Eq. 11).

7. Go to next time step.

\section{Results and discussion}

In this section, several numerical examples are solved. First the model for the droplets is validated in both two and three dimensions. Next, the wetting phenomena (contact of droplets and the substrate) are studied. Finally, analysis of droplet dynamics in PEFC gas channels is performed. Fluids' properties that have been used in the examples are displayed in Table 1 . These values correspond to a constant temperature of $298 \mathrm{~K}$ and a pressure of $1 \mathrm{~atm}$.

\footnotetext{
${ }^{2}$ For the examples solved in this work, the velocity of the droplet was not having considerable impact upon the airflow. Thus zero velocity can be used at $\Gamma_{\mathrm{I}}$.
}

Table 1 Water and air properties used in the simulations, considering $T=298 \mathrm{~K}$ and $p=1 \mathrm{~atm}$

\begin{tabular}{llll}
\hline Variable & Symbol & Value & Units \\
\hline $\begin{array}{l}\text { Surface tension } \\
\text { coefficient }\end{array}$ & $\gamma$ & 0.072 & $\mathrm{~N} \mathrm{~m}^{-1}$ \\
Water density & $\rho_{\mathrm{w}}$ & 1000 & $\mathrm{~kg} \mathrm{~m}^{-3}$ \\
Water viscosity & $\mu_{\mathrm{w}}$ & $10^{-3}$ & $\mathrm{~kg} \mathrm{~m}^{-1} \mathrm{~s}^{-1}$ \\
Air density & $\rho_{\text {air }}$ & 1.205 & $\mathrm{~kg} \mathrm{~m}^{-3}$ \\
Air viscosity & $\mu_{\text {air }}$ & $1.98 \times 10^{-5}$ & $\mathrm{~kg} \mathrm{~m}^{-1} \mathrm{~s}^{-1}$ \\
\hline
\end{tabular}

\subsection{Dynamic drop}

The free oscillations of a droplet are examined in this example. When its initial shape is different from the equilibrium one (i.e., spherical if gravity is neglected) the droplet exhibits several oscillations prior to reaching the equilibrium state. Lamb (see p. 475 in [26]) performed an analytical study and found the expression for droplets' eigenfrequencies:

$f_{n}=\frac{1}{2 \pi} \sqrt{\frac{n(n-1)(n+2) \gamma}{\rho R^{3}}}$

where $n$ is the oscillation mode, $\rho$ is the liquid's density, and $R$ is the droplet radius.

The considered setup is a water droplet placed in the center of a square domain filled with air. The initial shape of the droplet is elliptical (Fig. 3a), with $a=c=1$ and $b=3 \mathrm{~mm}$, as proposed in [3]. The density of water is set to $\rho=1000$ $\mathrm{kg} \mathrm{m}^{-3}$ in this example. The gravity force is neglected. Two different values of viscosity are used in order to check its effect on the resulting oscillations, and the time step is set to $10^{-5} \mathrm{~s}$ (this time step was suggested in [3]).

According to Eq. (25) and considering the first non-zero oscillation mode $(n=2)$, the frequency should be $\sim 43 \mathrm{~Hz}$. The values obtained using the present model $(f=46 \mathrm{~Hz}$ in $2 \mathrm{D}, f=42 \mathrm{~Hz}$ in 3D) show a very good agreement with the analytical value. The predicted frequency in $3 \mathrm{D}$ is closer to the actual value since it does not neglect the inertial effects in $z$-direction. The predicted value shows good agreement with the numerical simulation results from [3] as well $(\sim 50$ $\mathrm{Hz}$ ). Figure $3 \mathrm{c}, \mathrm{b}$ shows the $y$-displacement of an interface node $P$, with initial coordinates $(0,1,0) \mathrm{mm}$. Resulting displacement in the 3D example shows more oscillations than the 2D case. Since $z$ displacements are not taken into account in the 2D case, inertial effects in this direction are neglected as well. Figure 4 shows the evolution of the liquid domain over the simulation.

It is important to note that both the viscosity and the time step used in the numerical simulations have to be sufficiently low in order to observe oscillations in the droplet boundary [3]. No oscillations may be detected for large viscosity values, confirming the observation of Bouwhuis. 
Fig. 3 Shape and $y$-displacement evolution of an elliptic droplet with different viscosities, in two (right) and three (left) dimensions. a Initial and final droplet shapes;

b $y$-displacement of an interface node, $3 \mathrm{D}$ case; $\mathbf{c} y$-displacement of an interface node, $2 \mathrm{D}$ case
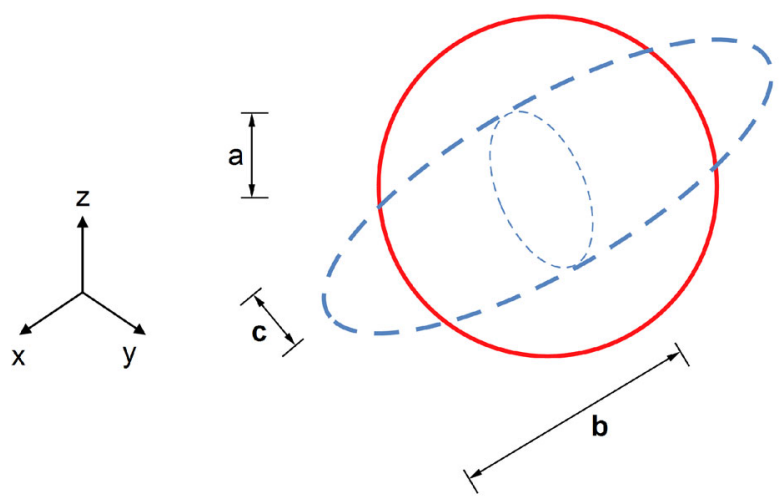

(a)

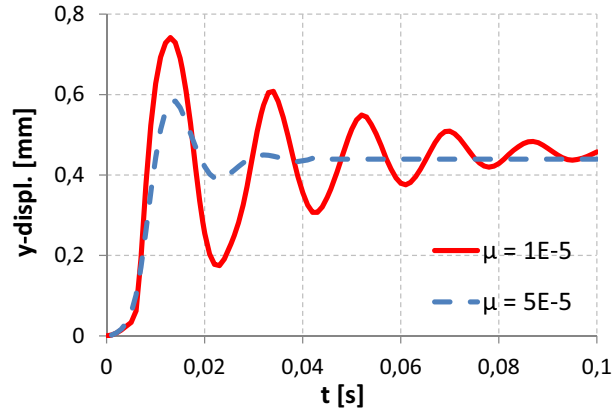

(b)

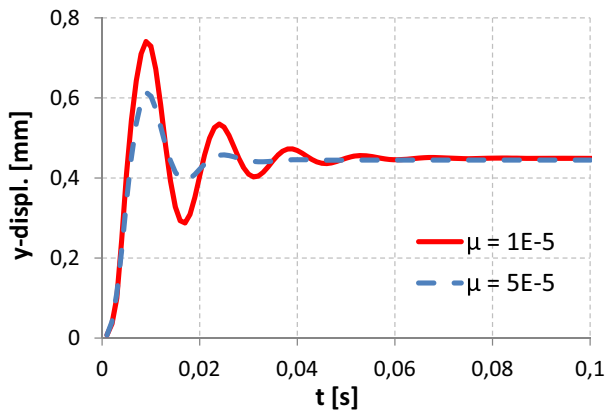

(c)

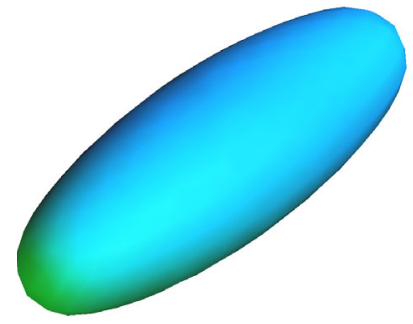

(a)

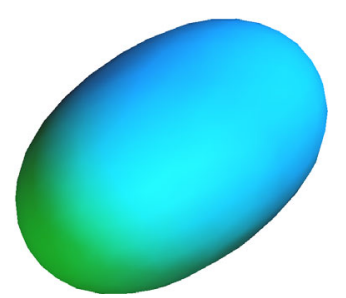

(c)

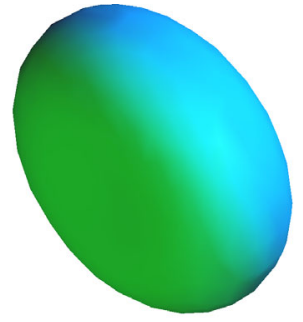

(b)

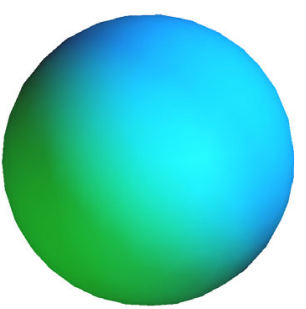

(d)
Fig. 4 Domain evolution for an elliptic drop with $\mu=10^{-5} \mathrm{~Pa} \mathrm{~s}$. a $t=0 \mathrm{~s} ; \mathbf{b} t=0.01 \mathrm{~s} ; \mathbf{c} t=0.025 \mathrm{~s} ; \mathbf{d} t=0.1 \mathrm{~s}$

\subsection{Sessile drop in different substrates}

When a droplet is placed on a solid substrate, three surface free energies are in equilibrium [20,21] $\gamma_{S L}$ (solid-liquid), $\gamma_{L G}$ (liquid-gas), and $\gamma_{S G}$ (solid-gas). At $\Gamma_{S}$ (Fig. 2), since curvature is zero, surface tension is also zero. At the contact line, the surface tension term is added using the normal vector corresponding to the static equilibrium configuration $\mathbf{n}_{e q}$ instead of the actual normal vector $\mathbf{n}$. More details on this condition can be found in reference [21].

The contact angle condition is validated in this example. A square of $1 \times 1 \mathrm{~mm}$ is set as the initial configuration. Triangular elements of $h=0.1 \mathrm{~mm}$ have been used to discretize the domain. Three different contact angles are taken into account: $\theta_{s}=70^{\circ}, 90^{\circ}$, and $135^{\circ}$. For the considered droplet size, gravitational effects can be neglected as given by the Bond number:

$B o=\frac{\rho g d^{2}}{\gamma}=\frac{1000 \times 9.81 \times\left(10^{-3}\right)^{2}}{0.072} \approx 0.14<1$

Results for the three different conditions are depicted in Fig. 5. Initially, the domain is represented by a square. Surface tension force acts on the interface $\Gamma_{\mathrm{I}}$, minimizing the curvature (i.e., the corners vanish). On the other hand, the until equilibrium is reached.

Table 2 shows the difference between the prescribed and the modeled contact angle. Obtained results show good agreement with the prescribed contact angle, giving a maximum relative error of $0.21 \%$ with a relatively coarse mesh. contact line (represented by two points) moves accordingly 
Fig. 5 Droplet evolution for three different contact angles.

a $\theta_{s}=70^{\circ}, t=0 \mathrm{~s}$;

b $\theta_{s}=90^{\circ}, t=0 \mathrm{~s}$;

c $\theta_{s}=135^{\circ}, t=0 \mathrm{~s}$;

d $\theta_{s}=70^{\circ}, t=0.1 \mathrm{~s}$;

e $\theta_{s}=90^{\circ}, t=0.1 \mathrm{~s}$;

f $\theta_{s}=135^{\circ}, t=0.1 \mathrm{~s}$

g $\theta_{s}=70^{\circ}, t=0.25 \mathrm{~s}$;

h $\theta_{s}=90^{\circ}, t=0.25 \mathrm{~s}$;

i $\theta_{s}=135^{\circ}, t=0.25 \mathrm{~s}$

$\mathbf{j} \theta_{s}=70^{\circ}, t=1 \mathrm{~s}$

$\mathbf{k} \theta_{s}=90^{\circ}, t=1 \mathrm{~s}$

$\mathbf{l} \theta_{s}=135^{\circ}, t=1 \mathrm{~s}$

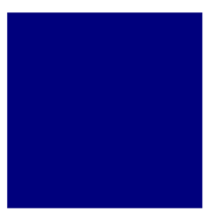

(a)

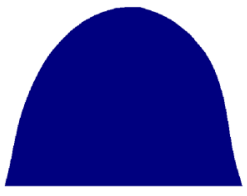

(d)

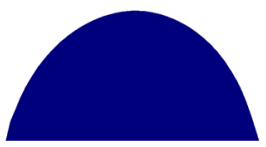

(g)

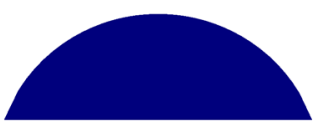

(j)

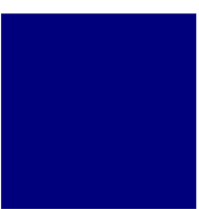

(b)

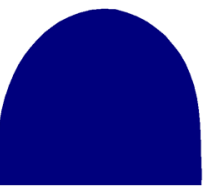

(e)

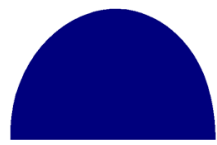

(h)

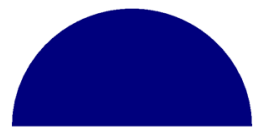

(k) (c)

(f)

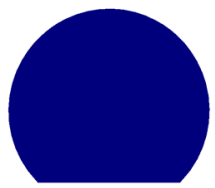

(i)
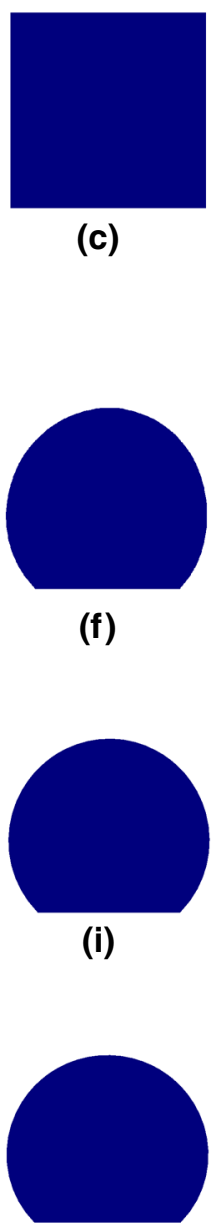

(I)
Table 2 Prescribed $\left(\theta_{s}\right)$ and obtained $\left(\theta_{\text {obs }}\right)$ contact angles, and relative error $\left(\epsilon_{\theta}\right)$ between these variables

\begin{tabular}{lccc}
\hline & $\theta_{s}=70^{\circ}$ & $\theta_{s}=90^{\circ}$ & $\theta_{s}=135^{\circ}$ \\
\hline$\theta_{\mathrm{obs}}\left[^{\circ}\right]$ & 70.15 & 89.91 & 135.08 \\
$\epsilon_{\theta}[\%]$ & 0.21 & -0.10 & 0.06 \\
\hline
\end{tabular}

\subsection{Injection}

In order to study water transport in PEFC gas channels and obtain results on droplet deformation, detachment, and GDL area coverage, which represent important factors for fuel cell performance and durability, water is injected from a circular pore in a gas channel. For a given air velocity, slug, droplet, or film flow can be observed depending on the injection rate of water, which, in turn corresponds to the current density. Slugs or films are observed for current densities i $>0.4 \mathrm{~A}$ $\mathrm{cm}^{-2}$ for the fuel cell gas channel considered in this work. Its geometry and properties are specified next.

The computational domain is a rectangular channel of $H \times$ $L$ in two dimensions (Fig. 6). Air enters into the channel

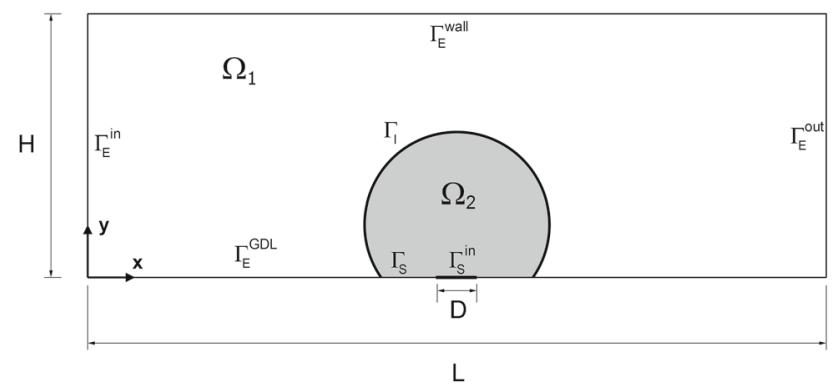

Fig. 6 Schematic representation of a water droplet in a PEFC gas channel in two dimensions

through the inlet, $\Gamma_{\mathrm{E}}^{\mathrm{in}}$, and flows in the positive x-direction. A no-slip boundary condition for air velocity is applied on the GDL surface, $\Gamma_{\mathrm{E}}^{\mathrm{GDL}}$, and zero viscous stress in the normal direction is imposed at the outlet $\Gamma_{\mathrm{E}}^{\text {out }}$ (i.e., the outlet of the channel is considered an open boundary). The channel has the sufficient length to ensure that results do not depend on this parameter.

A water droplet is considered to occupy the central position of the channel. The air-water interface is represented by 
Table 3 Parameters used in the droplet injection in a channel example

\begin{tabular}{llll}
\hline Variable & Symbol & Value & Units \\
\hline Initial chord & $c$ & 100 & $\mu \mathrm{m}$ \\
Water flow rate & $Q$ & 0.1 & $\mu 1 \mathrm{~s}^{-1}$ \\
Water inlet velocity & $\mathbf{v}_{\mathrm{w}}^{0}$ & 0.04 & $\mathrm{~m} \mathrm{~s}^{-1}$ \\
Static contact angle & $\theta_{\mathrm{S}}$ & 110 & $\circ$ \\
Air velocity & $\mathbf{v}_{\text {air }}^{0}$ & 10 & $\mathrm{~m} \mathrm{~s}^{-1}$ \\
Channel height & $H$ & 250 & $\mu \mathrm{m}$ \\
Channel width & $W$ & 250 & $\mu \mathrm{m}$ \\
Channel length & $L$ & 3 & $\mathrm{~mm}$ \\
Pore diameter & $D$ & 50 & $\mu \mathrm{m}$ \\
\hline
\end{tabular}

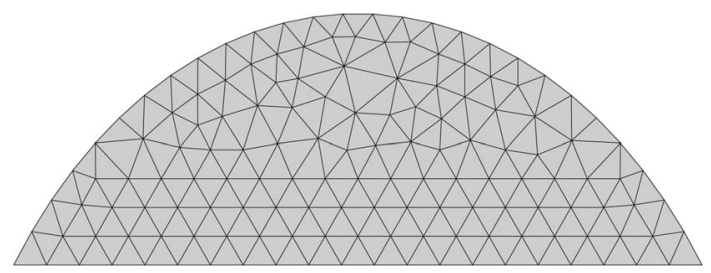

Fig. 7 Mesh used for the droplet injection example

$\Gamma_{\mathrm{I}}$ (Fig. 6), whereas $\Gamma_{S}$ denotes the wet area of the GDL (i.e., GDL surface covered by water). The center of $\Gamma_{S}$ contains the region $\Gamma_{S}^{\text {in }}$, representing a circular pore with diameter $D$. The distance between the pore and channel inlet is sufficiently big to avoid entrance effects [7] and air velocity profile is fully developed before the droplet's position. A slip boundary condition for water velocity is applied at $\Gamma_{S}$.

Wu and Djilali [37] performed an experiment to investigate the dynamics of water droplets emerging from a squared $50 \times 50 \mu \mathrm{m}$ pore into a $250 \times 250 \mu \mathrm{m}$ air channel. Although dimensions of the channel were different from a channel typically encountered in PEFCs, results provided valuable data on droplet deformation and detachment.
Channel was built using a transparent elastomer (polydimethylsiloxane, PDMS) with a static contact angle of $110^{\circ}$. This value is similar to that of carbon paper without PTFE coating [37]. Different flow regimes were considered, with Reynolds numbers ranging from 50 to 1200 . Droplet flow was obtained for an air velocity of $10 \mathrm{~m} \mathrm{~s}^{-1}$ and water volume flow of $0.1 \mu \mathrm{l} \mathrm{s}^{-1}$. Inlet velocity of water was $0.04 \mathrm{~m} \mathrm{~s}^{-1}$.

This example reproduces the experimental results from reference [37] to validate the numerical model. Boundary conditions for the air and water are the following:

- Dirichlet boundary condition at $\Gamma_{\mathrm{E}}^{\text {in }}$ with $\mathbf{v}_{\text {air }}=\mathbf{v}_{\text {air }}^{0}$ - No-slip boundary condition at $\Gamma_{\mathrm{E}}^{\mathrm{GDL}}$ and $\Gamma_{\mathrm{E}}^{\text {wall }}, \mathbf{v}_{\text {air }}=0$

- Dirichlet boundary condition at $\Gamma_{S}^{\text {in }}$ with $\mathbf{v}_{\mathrm{w}}=\mathbf{v}_{\mathrm{w}}^{0}$

Channel size as well as flow parameters are detailed in Table 3. Channel geometry has been discretized using an unstructured mesh of 25000 triangular elements. The mesh has been refined in the region of the channel where the pore and the emerging droplet are placed. Element sizes range from $h=5 \times 10^{-5} \mathrm{~m}$ to $h=5 \times 10^{-6} \mathrm{~m}$. Droplet mesh is unstructured with 200 triangular elements (see Fig. 7). Element size is constant with $h=5 \times 10^{-6}$. Simulations have been performed with a time step $\delta t=10^{-6} \mathrm{~s}$.

Lagrangian inlet In order to simulate water injection into the droplet, appropriate boundary conditions must be prescribed. In fixed-grid Eulerian models inlet conditions, such as air entering the channel, are represented via Dirichlet boundary conditions simply by prescribing the fixed velocity value. However, an inlet condition applied to a moving Lagrangian mesh is not trivial. In this work, the water injection process is done by creating new nodes in a specific region of the boundary, as shown in Fig. 8.

Initially, the inlet region is represented by a set of nodes with a given initial velocity (colored in red in Fig. 8a). These

Fig. 8 Lagrangian inlet process in a channel. a $t_{0}, V=V_{0}$; b $t_{1}, V=V_{0}$;

c $t_{2}, V=V_{0}+\Delta V$

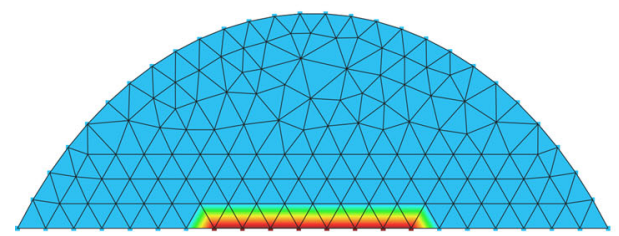

(a)

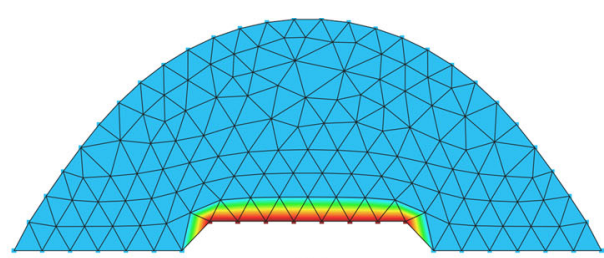

(b)

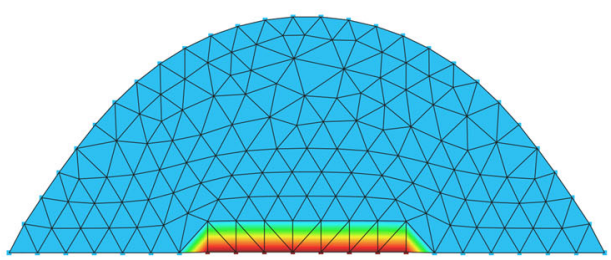

(c) 
nodes start to move according to the given velocity, leaving an empty space in the domain (Fig. 8b). The area occupied by this empty space is the same than the increase of area of the whole domain. After a certain time, when the distance from the nodes to the injection boundary is bigger than a prescribed value (computed as the distance between the two adjacent layers of nodes at the inlet on the initial mesh at $\mathrm{t}=0$, approximately equal to the height of the undeformed elements), a new set of nodes is created (Fig. 8c). Since the Lagrangian domain is discretized using the PFEM methodology, the re-meshing process introduces the new nodes in the updated mesh. This Lagrangian inlet method is advantageous for the problem at hand, since water can be considered an infinite reservoir. Note that there exist alternatives of modeling the inlet on moving grids, such as e.g., Arbitrary Lagrangian Eulerian technique that allows to maintain the inlet nodes fixed in an Eulerian fashion, while moving all the rest of the droplet domain nodes in a Lagrangian fashion.

In this section, the dynamics of an emerging droplet in a PEFC gas channel are studied. Water produced within the fuel cell emerges into the channel from the GDL pores. Depending on the operating conditions, water may evolve as a droplet, a slug, or a film. The following examples recreate these flow types.

\subsubsection{Droplet flow}

Experimental validation $\mathrm{Wu}$ performed an experimental study to observe effects of air and water inflow velocities on droplet dynamics [37]. The experimental setup was a rectangular channel of $250 \times 250 \mu \mathrm{m}$ cross-section and $3 \mathrm{~mm}$ length. Air entered the channel at a constant velocity of 10 $\mathrm{m} \mathrm{s}^{-1}$. Water was injected via a $50 \times 50 \mu \mathrm{m}$ pore with an inlet velocity of $0.04 \mathrm{~m} \mathrm{~s}^{-1}$. Wu observed that emergence and posterior detachment process was repeated every 0.075 ms approximately, giving a frequency $f=13.2 \mathrm{~Hz}$ (i.e., approximately 13 droplets per second emerged and detached from the pore). Experimental results are used to validate the numerical model.

Figure 9 depicts the process of water injection into a gas channel. Experimental results obtained in [37] are displayed in left-hand side column. Right-hand side column shows the predicted droplet profile according to the embedded method. Channel walls are represented by black lines, whereas the blue line is the droplet profile. The gap in the channel surface is the pore where water is injected. Predicted profiles are similar to those observed in [37], specially at the beginning of the injection process.

Evolution of contact advancing and receding angles according to Wu is shown in Fig. 10. Three regions can be distinguished: (I) both advancing and receding angles increase at the same rate, (II) receding angle remains constant, and (III) receding angle decreases at a constant rate.

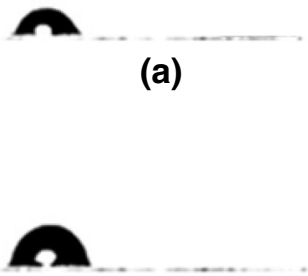

(c)

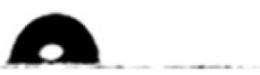

(e)

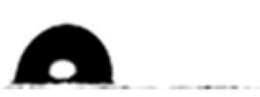

(g)

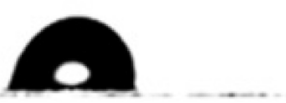

(i)

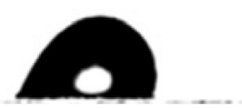

(k)

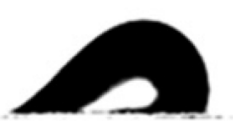

(m)

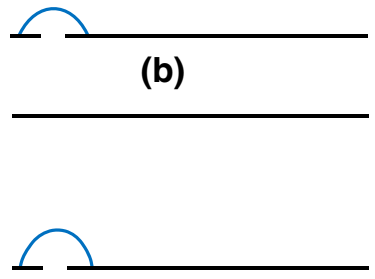

(d)

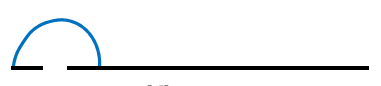

(f)

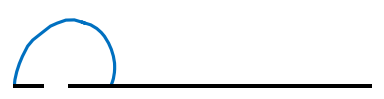

(h)

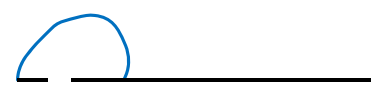

(j)

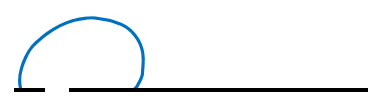

(I)

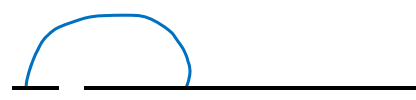

(n)
Fig. 9 Experimental (left column) and simulated (right column) deformation process of an emerging droplet into a gas channel. Experimental results extracted from [37]. a Experiment, $t=5 \mathrm{~ms}$; b Simulation, $t=5$ ms; c Experiment, $t=10 \mathrm{~ms}$; d Simulation, $t=10 \mathrm{~ms}$; e Experiment, $t=15 \mathrm{~ms} ; \mathbf{f}$ Simulation, $t=15 \mathrm{~ms} ; \mathbf{g}$ Experiment, $t=20 \mathrm{~ms} ; \mathbf{h}$ Simulation, $t=20 \mathrm{~ms}$; i Experiment, $t=25 \mathrm{~ms}$; j Simulation, $t=25$ $\mathrm{ms}$; k Experiment, $t=45 \mathrm{~ms}$; l Simulation, $t=45 \mathrm{~ms}$; $\mathbf{m}$ Experiment, $t=65 \mathrm{~ms} ; \mathbf{n}$ Simulation, $t=5 \mathrm{~ms}$

Numerical results show good agreement with experimental data from reference [37], particularly prior to the onset of droplet detachment. At early stages of droplet emergence (regions I and II), both predicted and measured contact angles have an excellent fit. After time $t=0.02 \mathrm{~s}$, the results obtained with the present numerical start over-predicting the 


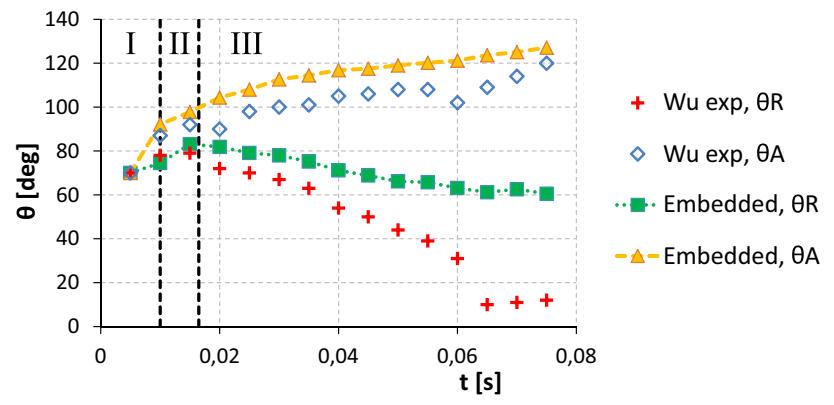

Fig. 10 Advancing (blue diamond markers) and receding angles observed in [37]. (Color figure online)

angles. Moreover, the difference between results is probably due to significant effects in the $z$ direction that start manifesting as the droplet deforms from a nearly axisymmetric shape, which is not considered in the 2D model.

At $t=0.045 \mathrm{~s}$ the droplet starts to break up from water stream injected through the pore. At $t>0.06$ the break-up effects become completely pronounced in the experiment (see the "neck" developed at the receding side). Whereas the advancing point is moving, the receding point is pinned. A model that includes droplet break-up effects must be developed in order to improve these discrepancies observed with respect to the experimental observations. This puts in evidence the importance of developing a fully three-dimensional model of droplet-airflow interaction, accounting for the deformation of the entire contact line (which cannot be done with a $2 \mathrm{D}$ model).

\section{Summary and conclusions}

The PFEM has been used to model surface tension-dominated droplet problems. The droplet model has been validated in both two and three dimensions. In two dimensions, the PFEM model was coupled to an Eulerian formulation for air to study droplet-airflow interactions. The model was capable of accurately including surface tension effects present at the dropletair interface. Wetting phenomena have also been analyzed, and the model can be used to simulate sessile droplets in substrates with variable contact angles. Droplet dynamics considering varying volumes have been experimentally validated and results show good agreement with available data. Analysis of post-detachment behavior defines an important task to be undertaken in future. Moreover, fully threedimensional simulations of droplet-airflow interaction have to be performed to accurately account for contact line deformations (which cannot be considered in a 2D model, where the contact line becomes represented by two triple points).

\section{Compliance with ethical standards}

Funding This work was supported under the auspices of the FPDI2013-18471 and BES-2011-047702 grants of the Spanish Ministerio de Economia y Competitividad as well as partially funded by the COMETAD project of the National RTD Plan (ref. MAT2014-60435$\mathrm{C} 2-1-\mathrm{R})$ of the mentioned ministry.

Conflict of interest The authors declare that they have no conflict of interest.

\section{References}

1. Akhtar N, Kerkhof PJAM (2011) Dynamic behavior of liquid water transport in a tapered channel of a proton exchange membrane fuel cell cathode. Int J Hydrog Energy 36(4):3076-3086

2. Bird R, Stewart W, Lightfoot E (2002) Transport phenomena, 2nd edn. Wiley, Hoboken

3. Bouwhuis W, Winkels KG, Peters IR, Brunet P, van der Meer D, Snoeijer JH (2013) Oscillating and star-shaped drops levitated by an airflow. Phys Rev E 88:023017

4. Brennen CE (2005) Fundamentals of multiphase flow, 1st edn. Cambridge University Press, Cambridge

5. Carton J, Lawlor V, Olabi A, Hochenauer C, Zauner G (2012) Water droplet accumulation and motion in PEM (Proton Exchange Membrane) fuel cell mini-channels. Energy 39:63-73

6. Chen K, Hickner M, Noble D (2005) Simplified models for predicting the onset of liquid water droplet instability at the gas diffusion layer/gas flow channel interface. Int J Energy Res 29(12):11131132

7. Cho SC, Wang Y, Chen K (2012) Droplet dynamics in a polymer electrolyte fuel cell gas flow channel: forces, deformation, and detachment. I: theoretical and numerical analyses. J Power Sources 206:119-128

8. Choi J, Son G (2009) Numerical study of droplet dynamics in a PEMFC gas channel with multiple pores. J Mech Sci Technol 23(7):1765-1772

9. Chorin AJ (1967) A numerical method for solving incompressible viscous problems. J Comput Phys 2:12-26

10. Codina R (2001) A stabilized finite element method for generalized stationary incompressible flows. Comput Methods Appl Mech Eng 190 (20-21):2681-2706. http://www.sciencedirect. com/science/article/pii/S0045782500002607 doi:10.1016/ S0045-7825(00)00260-7

11. Codina R (2001) Pressure stability in fractional step finite element method for incompressible flows. J Comput Phys 170:112-140

12. Crowe CT (2006) Multiphase flow handbook, 1st edn. Taylor \& Francis, Abingdon

13. Feng Y, Idelsohn S, Nigro N, Gimenez J, Rossi R, Marti J (2013) A fast and accurate method to solve the incompressible NavierStokes equations. Eng Comput 30(2):197-222

14. Ferreira RB, Falcão DS, Oliveira VB, Pinto AMFR (2015) Numerical simulations of two-phase flow in proton exchange membrane fuel cells using the volume of fluid method - A review. J Power Sources 277:329-342

15. Gerstenberger A, Wall W (2008) An extended finite element/Lagrange multiplier based approach for fluid-structure interaction. Comput Methods Appl Mech Eng 197:1699-1714

16. Gerstenberger A, Wall W (2010) An embedded Dirichlet formulation for 3D continua. Int J Numer Methods Eng 82(5):537-563

17. Gopala V, van Wachem G (2008) Volume of fluid methods for immiscible-fluid and free-surface flows. Chem Eng J 141:204-221

18. Guermond J, Minev P, Shen J (2006) An overview of projection methods for incompressible flows. Comput Methods Appl Mech Eng 195:6011-6045

19. Idelsohn SR, Oñate E, Pin FD (2004) The particle finite element method: a powerful tool to solve incompressible flows with free- 
surfaces and breaking waves. Int J Numer Methods Eng 61(7):964989

20. Israelachvili J (2011) Intermolecular and surface forces, 3rd edn. Elsevier, Waltham

21. Jarauta A, Ryzhakov PB, Secanell M, Waghmare PR, Pons-Prats J (2015) Numerical study of droplet dynamics in a Proton Exchange Fuel Cell gas channel using an embedded formulation. J Power Sources (submitted)

22. Jarauta A, Secanell M, Pons-Prats J, Ryzhakov PB, Idelsohn SR, Oñate E (2015) A semi-analytical model for droplet dynamics on the GDL surface of a PEFC electrode. Int J Hydrog Energy 40:5375-5383

23. Kamran K, Rossi R, Onate E, Idelsohn S (2013) A compressible Lagrangian framework for the simulation of the underwater implosion of large air bubbles. Comput Methods Appl Mech Eng 255:210-225

24. Kandlikar S, Lu Z, Domigan W, White A, Benedict M (2009) Measurement of flow maldistribution in parallel channels and its application to ex-situ and in-situ experiments in pemfc water management studies. Int J Heat Mass Transf 52(7):1741-1752

25. Küttler U, Wall W (2009) Vector extrapolation for strong coupling fluid-structure interaction solvers. J Appl Mech 76(2):021-205

26. Lamb H (1916) Hydrodynamics, 4th edn. Cambridge University Press, Cambridge. https://books.google.es/books?id=d_ AoAAAAYAAJ

27. Marti J, Ryzhakov P, Idelsohn S, Oñate E (2012) Combined Eulerian-PFEM approach for analysis of polymers in fire situations. Int J Numer Methods Eng 92:782-801

28. Mier-Torrecilla, M (2010) Numerical simulation of multi-fluid flows with the Particle Finite Element Method. Ph.D. thesis, Universitat Politécnica de Catalunya

29. Oñate E, Idelsohn S, del Pin F, Aubry R (2004) The particle finite element method: an overview. Int J Comput Methods 1:267-307
30. Ryzhakov P, Oñate E, Rossi R, Idelsohn S (2010) Lagrangian FE methods for coupled problems in fluid mechanics. CIMNE. ISBN: 978-84-96736-97-9

31. Ryzhakov PB, Jarauta A (2015) An embedded approach for immiscible multi-fluid problems. Int J Numer Methods Fluids. doi:10. $1002 /$ fld 4190

32. Sussman M, Ohta M (2009) A stable and efficient method for treating surface tension in incompressible two-phase flow. SIAM J Sci Comput 31:2447-2471

33. Temam R (1969) Sur l'approximation de la solution des equations de Navier-Stokes par la methode des pase fractionaires. Arch Ration Mech Anal 32:135-153

34. Theodorakakos A, Ous T, Gavaises M, Nouri J, Nikolopoulos N, Yanagihara H (2006) Dynamics of water droplets detached from porous surfaces of relevance to PEM fuel cells. J Colloid Interface Sci 300:673-687

35. Weber AZ, Borup RL, Darling RM, Das PK, Dursch TJ, Gu W, Harvey D, Kusoglu A, Litster S, Mench MM, Mukundan R, Owejan JP, Pharoah JG, Secanell M, Zenyuk IV (2014) A critical review of modeling transport phenomena in Polymer-Electrolyte fuel cells. J Electrochem Soc 161(12):F1254-F1299

36. Wörner M (2012) Numerical modeling of multiphase flows in microfluidics and micro process engineering: a review of methods and applications. Microfluid Nanofluid 12:841-886

37. Wu T, Djilali N (2012) Experimental investigation of water droplet emergence in a model polymer electrolyte membrane fuel cell microchannel. J Power Sources 208:248-256

38. Zhu X, Sui P, Djilali N (2008) Three-dimensional numerical simulations of water droplet dynamics in a PEMFC gas channel. J Power Sources 181:101-115 